\title{
Digital Petri Dish for On-chip Cell Monitoring
}

\author{
Guoan Zheng, Seung Ah Lee, Xiaoze Ou and Changhuei Yang \\ Department of Electrical Engineering, California Institute of Technology, Pasadena, CA \\ 91125, USA \\ gazheng@caltech.edu
}

\begin{abstract}
We report a digital Petri dish platform for on-chip cell monitoring. We demonstrate the ability to image confluent cell cultures with $6 \mathrm{~mm} \times 4 \mathrm{~mm}$ filed-of-view and $\sim 0.7 \mu \mathrm{m}$ resolution by using the proposed platform.

OCIS codes: (170.0110) Imaging system; (170.1530) Cell analysis.
\end{abstract}

\section{Introduction}

The digital Petri dish solution [1], terms ePetri, is a chip-scale lensfree microscopy platform that can automatically perform high resolution ( $\sim 0.7$ micron) microscopy imaging over a large field-of-view $(6 \mathrm{~mm} \times 4 \mathrm{~mm})$. Unlike the other lensless microscopy methods [2-4], this new approach is fully capable of working with cell cultures or any samples in which cells/bacteria may be contiguously connected, and thus, it can significantly improve Petri dish based cell/bacteria culture experiments. With this approach providing a compact, low-cost and disposable microscopy imaging solution, we can start to transit Petri dish based experiments from the traditionally laborintensive process to an automated and streamlined process. This technological shift from an inert petri-dish to an digital Petri dish platform is appropriately timely as well, because, the cost of high performance CMOS imaging sensors (which are widely used in cellphone cameras and webcams) have recently reached a price point where they can be used as recyclable or disposable components.

Conceptually, this method of microscopy imaging is simple to understand. We simply culture cells or place cells of interest directly on the surface of a CMOS image sensor, as shown in Fig. 1(a). We sequentially tilt/shift an incoherent illumination source above the sample and acquire a sequence of raw images. With the incremental tilt/shift of the illumination, the target cells' shadow will incrementally shift across the sensor pixels. The amount of shadow shift is proportional to the passivation layer thickness and the tilt/shift extent of the light source. As long as the shadow shift between each raw image frame is much smaller than the physical pixel size, we can then combine the information from multiple sub-pixel-shifted low resolution shadow images to create a single high resolution image with a pixel super resolution algorithm.

\section{The prototype setup of the giga-pixel microscopy system}

Our digital Petri dish prototype is shown in Fig. 1. This prototype was built on a commercial available monochrome CMOS image sensor with a $6 \mathrm{~mm}^{*} 4 \mathrm{~mm}$ imaging area filled with 2.2 micron pixels (Aptina MT9P031). The microlens layer and color filter on the sensor surface were removed to provide us with direct access to the sensor pixels. We glued a home-made square plastic well to the image sensor with poly-dimethylsiloxane (PDMS). We then used a thin PDMS layer $(\sim 100 \mu \mathrm{m})$ as a cover for this prototype. The thin PDMS layer served to prevent excessive evaporation of the culture media while allowing for $\mathrm{CO}_{2}$ exchange between the well and exterior. For illumination, we used the LED screen of a smartphone as the scanning illumination light source, as shown in Fig. 1(b-c). A holder was built with Lego building blocks to house the image sensor socket board and the smartphone. The screen of smartphone was set at about $2.0 \mathrm{~cm}$ away from the image sensor. In this method, the alignment between the smartphone and the image sensor is not a critical consideration. The entire platform can be placed in an incubator for automatic long term cell imaging and tracking.

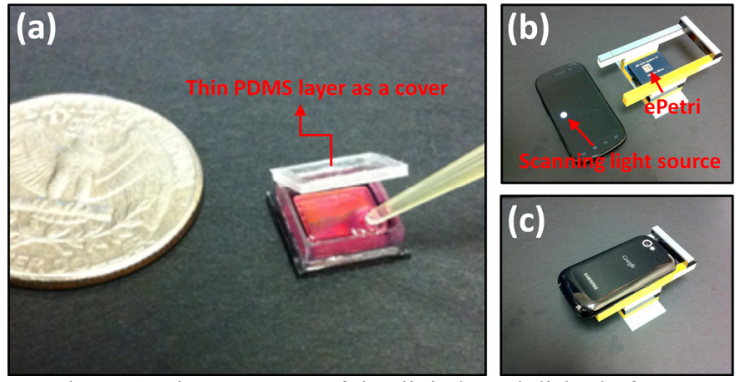

Figure 1. The prototype of the digital Petri dish platform. 


\section{Results}

Fig. 2(a) shows the reconstructed color image of the confluent HeLa cell sample. The amount of details in the reconstructed color image is too large to fully display on a computer screen or print on a printer; we have provided vignette views of selected regions for comparison in Fig. 2. Fig. 2(b1) and (c1) shows the raw images from a small region of Fig. 2(a). Fig. 2(b2) and (c2) show the corresponding reconstructed high resolution image of (b1) and (c1). From the reconstructed high resolution image in Fig. 2(b2) and (c2), we can readily discern organelles within the HeLa cell, such as multiple nuclear granules (indicated by red arrows), and the nucleus.

The digital Petri dish is a platform technology. Since the top surface of the sensor chip is unmodified, a user is free to build upon it. It is very possible to simply use the digital Petri dish as an imaging platform for a large number of sophisticated lab-on-a-chip designs, such as microorganisms detection based on the use of closed dielectrophoretic cages, droplet-based platforms for cell encapsulation and screening, microfluidics-based phenotyping imaging and screening of multicellular organisms, and high throughput malaria infected erythrocyte separation and imaging. It is also possible to incorporate other imaging functionalities, such as 3D and darkfield imaging $[5,6]$, into proposed digital Petri dish platform.

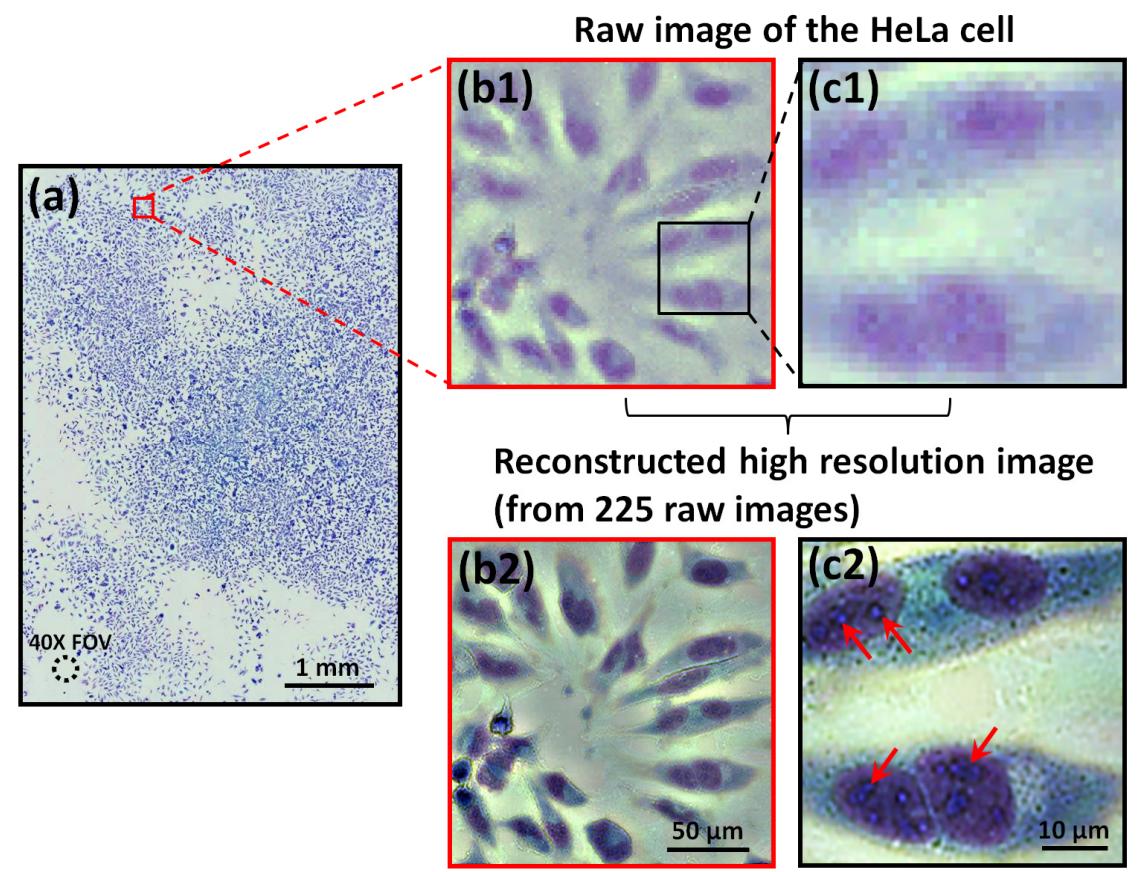

Figure 2 (a) Large-field-of-view color imaging of the confluent cell sample. The field of view of a 40X objective lens is also shown in left bottom. (b1) and (c1), raw images of a small region of (a). (b2) and (c2), the reconstructed high resolution images corresponding to (b1) and (c1). From (c2), we can readily discern organelles within the HeLa cell, such as multiple nuclear granules (indicated by red arrows), and the nucleus.

\section{References}

[1] G. Zheng, S. A. Lee, Y. Antebi, M. B. Elowitz, and C. Yang, "The ePetri dish, an on-chip cell imaging platform based on subpixel perspective sweeping microscopy (SPSM)," Proceedings of the National Academy of Sciences, vol. 108, pp. 16889-16894, 2011.

[2] O. Mudanyali, D. Tseng, C. Oh, S. O. Isikman, I. Sencan, W. Bishara, C. Oztoprak, S. Seo, B. Khademhosseini, and A. Ozcan, "Compact, light-weight and cost-effective microscope based on lensless incoherent holography for telemedicine applications," Lab on a Chip, vol. 10, p. $1417,2010$.

[3] G. Zheng, S. A. Lee, S. Yang, and C. Yang, "Sub-pixel resolving optofluidic microscope for on-chip cell imaging," Lab on a Chip, vol. 10, pp. 3125-3129, 2010.

[4] W. Xu, M. Jericho, I. Meinertzhagen, and H. Kreuzer, "Digital in-line holography for biological applications," Proceedings of the National Academy of Sciences, vol. 98, p.11301, 2001.

[5] G. Zheng, C. Kolner, and C. Yang, "Microscopy refocusing and dark-field imaging by using a simple LED array," Optics letters 36, 3987$3989,2011$.

[6] G. Zheng, X. Cui, and C. Yang, "Surface-wave-enabled darkfield aperture for background suppression during weak signal detection," Proceedings of the National Academy of Sciences, vol. 107, pp. 9043-9049, 2010. 